\title{
Toeplitz Operators on Higher Cauchy-Riemann Spaces Over the Unit Ball
}

\author{
Lijia Ding and Kai Wang
}

\begin{abstract}
In this paper, we investigate some algebraic properties of Toeplitz operators over higher Cauchy-Riemann spaces $C_{\alpha, m}$ on the unit ball $\mathbb{B}^{d}$ with $d \geq 2$. We first discuss the Berezin transform on higher Cauchy-Riemann spaces. By making use of Berezin transform, we completely characterize (semi-)commuting Toeplitz operators with bounded pluriharmonic symbols over higher Cauchy-Riemann space $C_{\alpha, m}$. Moreover, we show that compact products of finite Toeplitz operators with a class of bounded pluriharmonic symbols only happen in the trivial case.
\end{abstract}

Mathematics Subject Classification. Primary 46E22; Secondary 47B35.

Keywords. Berezin transform, $\mathcal{M}$-harmonic function, Higher CauchyRiemann space, Toeplitz operator, Pluriharmonic function.

\section{Introduction}

This paper is an attempt to study Toeplitz operators theory over higher Cauchy-Riemann spaces on bounded symmetric domain of complex spaces. While there exist abundant works on function theory for higher CauchyRiemann spaces, investigation of the operator aspect was started only a few years.

Let us start by recalling a few basic facts and some terminologies. Let $\Omega$ be an irreducible bounded symmetry domain, and $G=\operatorname{Aut}(D)$ the biholomorphic automorphism group. It is well known $[9,11]$ that via change of variables, the group $G$ unitarily acts on a series of weighted Bergman spaces $L_{a}^{2}\left(\Omega, d \mu_{\alpha}\right)$ with $d \mu_{\alpha}=h(z, z)^{\alpha} d m(z), \alpha>-1$, here $h(z, w)$ is a sesquiholomorphic polynomial called the quasi-determinant. Indeed it may quickly follow by that the fact $L_{a}^{2}\left(\Omega, d \mu_{\alpha}\right)=\operatorname{ker} \bar{D} \cap L^{2}\left(\Omega, d \mu_{\alpha}\right)$, where $\bar{D}$ is the invariant Cauchy-Riemann operator on $\Omega$. We now denote by $C_{\alpha, m}(\Omega)=$ $\operatorname{ker} \bar{D}^{m+1} \cap L^{2}\left(\Omega, d \mu_{\alpha}\right)$ the $m$ th Cauchy-Riemann space. Those $G$-invariant spaces, called as nearly holomorphic function spaces, was first studied by Shimura. In the paper [16], he found some important connections between nearly holomorphic functions and nonholomorphic automorphic forms in

The project was partially supported by NSFC (11722102), the Alexander von Humboldt Foundation (1151823), Shanghai Pujiang Program(16PJ1400600). 
Kähler manifold. Lately Engliš, Peetre, Peng and Zhang made a thorough study on them via the invariant Cauchy-Riemann operator $\bar{D}$. We refer the readers to $[6,7,12,13,18]$ for more details. These results reveal a beautiful relationship among Kähler geometry, Lie group representation theory and classical holomorphic function theory.

We now turn to operator aspect of higher Cauchy-Riemann spaces. It is natural to expect the structure of Toeplitz operators over higher CauchyRiemann spaces reveal a rich function theory picture directly dependent on the symbol of the Toeplitz operator and geometry of symmetric domains. One such instance has been made by Engliš and Zhang [8]. In their breakthrough, they discuss Toeplitz operators on the higher Cauchy-Riemann space $C_{\alpha, m}$ and established an interesting Dixmier trace formula involving pseudodifferential symbols and geometry of the boundary.

Initiated by a seminal work of Brown and Halmos [2], the study of algebraic properties for Toeplitz operators has inspired much deep researches and prompted many interesting problems on the classical Hardy space $H^{2}(\mathbb{D})$, the classical Bergman space $L_{a}^{2}(\mathbb{D})$ or others; see, for example, $[19,20]$ and the references therein. We will attempt to start an systematic study in higher Cauchy-Riemann spaces setting. In the present paper we will begin our study with the case of the unit ball $\mathbb{B}^{d}$, the bounded symmetric domain of rank 1 . It will be shown that some results in classical Bergman space $L_{a}^{2}(\mathbb{D})$ of the unit disc $\mathbb{D}$ have free analogues in higher Cauchy-Riemann spaces $C_{\alpha, m}\left(\mathbb{B}^{d}\right)$ setting.

To ease the notation, we write $C_{\alpha, m}=C_{\alpha, m}\left(\mathbb{B}^{d}\right)=k e r \bar{D}^{m+1} \cap L^{2}\left(\mathbb{B}^{d}\right.$, $\left.d \mu_{\alpha}\right)$ for some nonnegative integer. Some careful arguments of their function theory have appeared in the paper of Zhang and his colleague $[13,17,18]$. In particular, one sees that $C_{\alpha, m}=C_{\alpha, D(\alpha)}$ whenever $m \geq D(\alpha)$; see the definition $D(\alpha)$ in Eq. 2.1. Therefore, from now on we will always assume that $m$ is a fixed integer such that $0 \leq m \leq D(\alpha)$. We first introduced Berezin transform in the $C_{\alpha, m}$ setting. It is proved that Berezin transform $B_{\alpha, m}$ on $C_{\alpha, m}$ can be written a finite linear combination of some usual weighted Berezin transforms. We also show a fixed point theorem for Berezin transform. With its help we completely characterize when Toeplitz operators with bounded pluriharmonic symbols commute. More precisely, $T_{f} T_{g}=T_{g} T_{f}$ for bounded pluriharmonic functions $f, g$ if and only if one of the following holds:

(1) Both $f$ and $g$ are holomorphic.

(2) Both $\bar{f}$ and $\bar{g}$ are holomorphic.

(3) Either $f$ or $g$ is constant on $\mathbb{B}^{d}$.

(4) There is a nonzero constant $b$ such that $f-b g$ is constant on $\mathbb{B}^{d}$.

We also characterize semi-commuting Toeplitz operators. We final turn to the compact properties. It is proved that there aren't nontrivial compact Toeplitz operators with bounded pluriharmonic symbols on $C_{\alpha, m}$. We also consider the problems of compact product of finite Toeplitz operators with bounded pluriharmonic symbols. Suppose that all bounded functions $u_{i}$ have continuous extensions to some common nonempty relatively open subset of the boundary. We proved that in this case, the product of Toeplitz operators 
$T_{u_{i}}$ is compact if and only if at least one of $u_{i}$ is zero. For general symbols, the problem still remains a mystery.

The paper is organized as follows. In Sect. 2, we recall some necessary background and give an explicit description of higher Cauchy-Riemann spaces. In Sect. 3, we introduce the Berezin transform and show a fixed point theorem. We next characterize (semi-)commuting Toeplitz operators with bounded pluriharmonic symbol functions on $C_{\alpha, m}$ in Sect. 4. Finally, in Sect. 5 we will discuss compact products of finite Toeplitz operators with bounded pluriharmonic symbol functions.

\section{Preliminaries}

In this section we introduce some notations of the higher Cauchy-Riemann spaces on bounded symmetric domains; especially in the case of the unit ball, the bounded symmetric domain of rank 1 . We first recall the definition of the invariant Cauchy-Riemann operator.

Let $\Omega$ be a bounded symmetric domain with the Bergman kernel $K(z, w)$. It is well known that $\Omega$ has a Kähler metric given by the matrix $\left\{h_{i, \bar{j}}\right\}$, where $h_{i, \bar{j}}=\partial_{i} \bar{\partial}_{j} \log K(z, z)$. Then for a Hermitian vector bundle $E$ over $\Omega$, there exists an invariant Cauchy-Riemann operator

$$
\bar{D}_{E}: C^{\infty}(\Omega, E) \rightarrow C^{\infty}\left(\Omega, E \otimes T^{(1,0)}(\Omega)\right)
$$

involving this metric, where $T^{(1,0)}(\Omega)$ is the holomorphic tangent bundle of $\Omega$. Indeed, let $e_{\beta}$ be a collection of local trivializing sections of $E$. For a smooth section $f=f^{\beta} e_{\beta} \in C^{\infty}(\Omega, E)$, the operator $\bar{D}_{E}$ is given locally by

$$
\bar{D}_{E} f=\sum_{i, j} h^{\bar{j}, i} \frac{\partial f^{\beta}}{\partial \bar{z}^{j}} e_{\beta} \otimes \partial_{i},
$$

where $\left\{h^{\bar{j}, i}\right\}$ is the inverse of the matrix $\left\{h_{i, \bar{j}}\right\}$, and $\left\{\partial_{i}\right\}$ is the standard base of $T^{(1,0)}(\Omega)$. It follows the important invariance property of $\bar{D}_{E}$; namely, for a biholomorphism mapping $\varphi$ of $\Omega$,

$$
\bar{D}_{E}\left(U_{\varphi} f\right)=\left(U_{\varphi} \otimes(d \varphi)^{-1}\right) \bar{D}_{E} f,
$$

where $U_{\varphi}$ is the action on sections of $E$ induced by $\varphi$. Moreover, by iteration one may get the invariant operator $\bar{D}_{E}^{m}$ for any positive integer $m$, which is an operator such that

$$
\bar{D}_{E}^{m}: C^{\infty}(\Omega, E) \rightarrow C^{\infty}\left(\Omega, E \otimes\left(\otimes^{m} T^{(1,0)}(\Omega)\right)\right) .
$$

We refer the reader to $[6,8]$ for more details.

We now return to the case of unit ball $\mathbb{B}^{d}$ in complex space $\mathbb{C}^{d}$. Take $E$ the trivial bundle $E=\mathbb{C}$, then the smooth sections of $E$ are just smooth functions $C^{\infty}\left(\mathbb{B}^{d}, \mathbb{C}\right)$. For any $f \in C^{\infty}\left(\mathbb{B}^{d}, \mathbb{C}\right)$, the invariant Cauchy-Riemann operator $\bar{D}: C^{\infty}\left(\mathbb{B}^{d}, \mathbb{C}\right) \rightarrow C^{\infty}\left(\mathbb{B}^{d}, \mathbb{C} \otimes T^{(1,0)}\right)$ is given by

$$
\bar{D} f=\left(1-|z|^{2}\right)\left(\sum_{j=1}^{d} \frac{\partial f}{\partial \bar{z}^{j}} \otimes \partial_{j}-\sum_{j, k=1}^{d} z^{j} \bar{z}^{k} \frac{\partial f}{\partial \bar{z}_{k}} \otimes \partial_{j}\right) .
$$


By induction, we have the $\bar{D}^{m}: C^{\infty}\left(\mathbb{B}^{d}, \mathbb{C}\right) \rightarrow C^{\infty}\left(\mathbb{B}^{d}, \mathbb{C} \otimes^{m} T^{(1,0)}\right)$ is well defined for every positive integer $m$. The function in the space $\cup_{m=1}^{\infty}$ $\operatorname{ker} \bar{D}^{m}$ is called nearly holomorphic function in the sense of Shimura, see $[16]$.

For $\alpha>-1$, let $L^{2}\left(\mathbb{B}^{d}, d \mu_{\alpha}\right)$ be the $L^{2}$-integrable function space with respect to the weighted measure $d \mu_{\alpha}=\left(1-|z|^{2}\right)^{\alpha} d m(z)$, where $d m$ is the normalized Lebesgue measure on $\mathbb{B}^{d}$. Denote $C_{\alpha, m}=\operatorname{ker} \bar{D}^{m+1} \cap L^{2}\left(\mathbb{B}^{d}, d \mu_{\alpha}\right)$ be the $m$ th Cauchy-Riemann spaces, which are closed in $L^{2}\left(\mathbb{B}^{d}, d \mu_{\alpha}\right)$ by the fact that differential operators $\bar{D}^{m+1}$ are closed. By [16,18], $C_{\alpha, n}=C_{\alpha, D(\alpha)}$ whenever $n \geq D(\alpha)$, where

$$
D(\alpha)= \begin{cases}\frac{\alpha+1}{2}-1, & \alpha \text { is an odd integer }, \\ {\left[\frac{\alpha+1}{2}\right],} & \text { otherwise }\end{cases}
$$

where $\left[\frac{\alpha+1}{2}\right]$ is the integer part of $\frac{\alpha+1}{2}$. It also proved in $[13,18]$ that when $0 \leq m \leq D(\alpha)$,

$$
C_{\alpha, m}=\left\{\sum_{j=0}^{m} \sum_{|I|=j} \frac{\bar{z}^{I}}{\left(1-|z|^{2}\right)^{j}} g_{I}: g_{I} \in L_{a}^{2}\left(d \mu_{\alpha-2|I|}\right), \quad \forall I\right\} .
$$

Here for any mult-index $I$, write $|I|=\sum_{k=1}^{d} I_{k}, z^{I}=\prod_{k=1}^{d} z_{k}^{I_{k}}$. Then we immediately have the following relationship

$$
C_{\alpha, 0} \subset C_{\alpha, 1} \subset \cdots \subset C_{\alpha, D(\alpha)}=C_{\alpha, n}, \quad \forall n \geq D(\alpha) .
$$

By the definition, it is easy to check $C_{\alpha, m}$ is invariant under the action of any biholomorphism. More precisely, for every biholomorphism $\varphi \in A u t\left(\mathbb{B}^{d}\right)$, define a unitary action $U_{\varphi}$ on $L^{2}\left(\mathbb{B}^{d}, d \mu_{\alpha}\right)$ by

$$
U_{\varphi}(f)(z)=f(\varphi(z))\left(J_{\varphi}(z)\right)^{\frac{\alpha+d+1}{d+1}}, \quad \forall z \in \mathbb{B}^{d},
$$

where $J_{\varphi}(z)$ is the complex Jecobian of $\varphi$ at point $z$. By the invariant property of $\bar{D}$, we have that $U_{\varphi}\left(C_{\alpha, m}\right)=C_{\alpha, m}$. Clearly it yields a natural unitary representation of $A u t\left(\mathbb{B}^{d}\right)$ on $C_{\alpha, m}$.

Moreover, denote $A_{j}^{\alpha, 2}$ by the subspace of $L^{2}\left(\mathbb{B}^{d}, d \mu_{\alpha}\right)$ generated by $\triangle_{j}(z)=\left(\frac{\bar{z}_{1}^{1}}{1-|z|^{2}}\right)^{j}$ under the above action of $\operatorname{Aut}\left(\mathbb{B}^{d}\right), j=0, \ldots, D(\alpha)$; in other words, $A_{j}^{\alpha, 2}=\overline{\operatorname{span}}\left\{U_{\varphi} \triangle_{j}: \varphi \in \operatorname{Aut}\left(\mathbb{B}^{d}\right)\right\}$. It is shown that $A_{j}^{\alpha, 2}$, $j=0, \ldots, D(\alpha)$, are exactly all irreducible representations of $A u t\left(\mathbb{B}^{d}\right)$, and each of them is called discrete series of $L^{2}\left(\mathbb{B}^{d}, d \mu_{\alpha}\right)$ as in $[15,18]$. In particular, in the case $j=0$, we have that the subspace $A_{0}^{\alpha, 2}=L_{a}^{2}\left(\mathbb{B}^{d}, d \mu_{\alpha}\right)$ is the standard weighted Bergman space. With some arguments of representation theory, Zhang [17] showed the that for $0 \leq m \leq D(\alpha), C_{\alpha, m}$ has an irreducible orthogonal decomposition

$$
C_{\alpha, m}=\oplus_{j=0}^{m} A_{j}^{\alpha, 2}
$$

with respect to the action of $A u t\left(\mathbb{B}^{d}\right)$. This implies that the following holds. 
Proposition 2.1. The space $C_{\alpha, m}$ is a reproducing kernel space with the reproducing kernel

$\mathbf{K}_{(\alpha, m)}(z, w)=\frac{1}{(1-\langle z, w\rangle)^{(\alpha+d+1)}} \sum_{j=0}^{m} c(\alpha, j) \frac{|1-\langle z, w\rangle|^{2 j}}{\left(1-|z|^{2}\right)^{j}\left(1-|w|^{2}\right)^{j}}, \quad \forall z, w \in \mathbb{B}^{d}$

for some constant $c(\alpha, j)$.

Proof. It is [17] proved that each $A_{j}^{\alpha, 2}$ is a reproducing kernel space with the kernel

$$
\begin{aligned}
& K_{\alpha, j}(z, w) \\
& \quad=\frac{C_{j}}{(1-\langle z, w\rangle)^{\alpha+d+1}} \sum_{k=0}^{j} \frac{(-j)_{k}(j-\alpha-1)_{k}}{k ! d !}\left(1-\frac{|1-\langle z, w\rangle|^{2}}{\left(1-|z|^{2}\right)\left(1-|w|^{2}\right)}\right)^{k}
\end{aligned}
$$

with the constat

$$
C_{j}=\frac{\Gamma(d+j) \Gamma(D(\alpha)+1-j) \Gamma(\alpha+d+1)(\alpha+1-2 j)}{\Gamma(d) \Gamma(d+1) \Gamma(\alpha+2-j)(-1)^{j} \prod_{k=0, k \neq j}^{D(\alpha)}(k-j)},
$$

where $(\nu)_{k}=\Pi_{j=0}^{k-1}(\nu+j)$ denoted by the Pochhammer for any complex number $\nu$. This implies that $C_{\alpha, m}$ is also a reproducing kernel space with the kernel

$$
\mathbf{K}_{(\alpha, m)}(z, w)=\sum_{j=0}^{m} K_{\alpha, j}(z, w),
$$

which leads to the desired result by a direct computation.

For each bounded function $f \in L^{\infty}\left(\mathbb{B}^{d}\right)$, we define the Toeplitz operator $T_{f}^{(\alpha, m)}$ on $C_{\alpha, m}$ by

$$
T_{f}^{(\alpha, m)}(g)=P(f g), \quad \forall g \in C_{\alpha, m},
$$

where $P$ is the orthogonal projection from $L^{2}\left(\mathbb{B}^{d}, d \mu_{\alpha}\right)$ onto $C_{\alpha, m}$. In the following context, we write them by $T_{f}$ whenever no confusion arises. Obviously one has that

$$
T_{f} g(z)=\int_{\mathbb{B}^{d}} f(w) g(w) \mathbf{K}_{(\alpha, m)}(z, w) d \mu_{\alpha}(w), z \in \mathbb{B}^{d}, g \in C_{\alpha, m} .
$$

\section{Berezin Transform on $C_{\alpha, m}$}

In this section we will define Berezin transform on $C_{\alpha, m}$. We first recall Berezin transform on weighted Bergman space $L_{a}^{2}\left(\mathbb{B}^{d}, d \mu_{\alpha}\right)$. For a function $f \in L^{1}\left(\mathbb{B}^{d}, d \mu_{\alpha}\right)$, the Berezin transform of $B_{\alpha} f$ is given by

$$
B_{\alpha} f(z)=\left\langle f k_{\alpha, z}, k_{\alpha, z}\right\rangle=c_{\alpha} \int_{\mathbb{B}^{d}} f \circ \varphi_{z}(w) d \mu_{\alpha}(w), \quad z \in \mathbb{B}^{d} .
$$


Here $c_{\alpha}=\frac{\Gamma(d+\alpha+1)}{\Gamma(d+1) \Gamma(\alpha+1)}, k_{\alpha, z}(w)$ is the normalized reproducing kernel on $L_{a}^{2}\left(\mathbb{B}^{d}, d \mu_{\alpha}\right)$ which is given by

$$
k_{\alpha, z}(w)=\frac{\sqrt{c_{\alpha}}\left(1-|z|^{2}\right)^{\frac{d+\alpha+1}{2}}}{(1-\langle z, w\rangle)^{d+\alpha+1}}
$$

and $\varphi_{a}$ is the Möbius transform at the point $a \in \mathbb{B}^{d}$, see Sect. 2.2.1 of [14]. We note that $B_{\alpha} 1=1$, and $B_{\alpha} f$ is smooth inside the unit ball for any $f \in L^{1}\left(\mathbb{B}^{d}, d \mu_{\alpha}\right)$.

Now we turn to define Berezin transform on $C_{\alpha, m}$. From (2.4) and (2.6), we know that

$$
\mathbf{K}_{(\alpha, m)}(z, z)=\sum_{j=0}^{m} K_{\alpha, j}(z, z)=\frac{\sum_{j=0}^{m} C_{j}}{\left(1-|z|^{2}\right)^{\alpha+d+1}} .
$$

It follows that the normalized reproducing kernel at $z, w \in \mathbb{B}^{d}$ is given by

$$
\begin{aligned}
\mathbf{k}_{(\alpha, m), w}(z) & =\mathbf{k}_{(\alpha, m)}(z, w) \\
& =\frac{\left(1-|w|^{2}\right)^{\frac{\alpha+d+1}{2}}}{\sqrt{\sum_{j=0}^{m} C_{j}}(1-\langle z, w\rangle)^{(\alpha+d+1)}} \sum_{j=0}^{m} c(\alpha, j) \frac{|1-\langle z, w\rangle|^{2 j}}{\left(1-|z|^{2}\right)^{j}\left(1-|w|^{2}\right)^{j}} .
\end{aligned}
$$

For convenience, we introduce some notations. Denote by $B(X, Y)$ the set of all bounded operators from Banach space $X$ to Banach space $Y$, and we also use $B(X)$ instead of $B(X, X)$ as usual. Moreover, we write the function space $L^{p}\left(\mathbb{B}^{d}, d \mu_{\alpha}\right)$ in simplified form $L^{p}\left(d \mu_{\alpha}\right)$. Then we give the definition of Berezin transform on $C_{\alpha, m}$.

Definition 3.1. (1) For $f \in L^{1}\left(d \mu_{\alpha-2 m}\right)$, then the Berezin transform $B_{\alpha, m} f$ is given by

$$
B_{\alpha, m} f(z)=\left\langle f \mathbf{k}_{(\alpha, m), z}, \mathbf{k}_{(\alpha, m), z}\right\rangle, \quad z \in \mathbb{B}^{d} .
$$

(2) For $T \in B\left(C_{\alpha, m}\right)$, the Berezin transform $B_{\alpha, m}$ for $T$ is given by

$$
B_{\alpha, m} T(z)=\left\langle T \mathbf{k}_{(\alpha, m), z}, \mathbf{k}_{(\alpha, m), z}\right\rangle, \quad z \in \mathbb{B}^{d} .
$$

By definition, one sees that $B_{\alpha, m} T_{f}=B_{\alpha, m} f$ for any $f \in L^{\infty}\left(\mathbb{B}^{d}\right)$ as usual. However, the property for $B_{\alpha, m}$ becomes much compliant. For example, $B_{\alpha, m}$ is even not injective in general cases.

Example. Let $g=1$ and $f=\frac{\bar{z}^{\gamma}}{\left(1-|z|^{2}\right)^{|\gamma|}} z^{\gamma}$ for any multi-index $\gamma$ with $0<$ $|\gamma| \leq m$. Recall that $f \otimes g-g \otimes f$, defined by

$$
(f \otimes g-g \otimes f)(h)=\langle h, g\rangle f-\langle h, f\rangle g, \quad \forall h \in C_{a, m},
$$

is a finite rank operator of rank 2. But, a direct computation shows $B_{\alpha, m}(f \otimes$ $g-g \otimes f)=0$.

We next show that $B_{\alpha, m}$ has still a close relation with the Berezin transform $B_{\alpha}$ over the weighted Bergman space $L_{a}^{2}\left(\mathbb{B}^{d}, d \mu_{\alpha}\right)$. We first notice that $B_{\alpha-j}$ belongs to the set $B\left(L^{1}\left(d \mu_{\alpha-2 m}\right), L^{1}\left(d \mu_{\alpha-2 m+1}\right)\right)$ for $0 \leq j \leq$ $2 m$, by Proposition 1.4.10 of [14]. For our purpose, we give the definition as following. 
Definition 3.2. A bounded operator $A \in B\left(L^{1}\left(d \mu_{\alpha-2 m}\right), L^{1}\left(d \mu_{\alpha-2 m+1}\right)\right)$ is called a Berezin transform sum operator, if there are some real constants $\mu_{0}, \ldots, \mu_{2 m}$ satifying $\sum_{j=0}^{2 m} \mu_{j}=1$ such that

$$
A=\sum_{j=0}^{2 m} \mu_{j} B_{\alpha-j} .
$$

This type operaor has been consider by Lee [10] in the case of nonnegative coefficients. We first remark the following obersevation.

Lemma 3.3. For a Berezin transform sum operator $A$, then the representation in Definition 3.2 for $A$ is unique.

Proof. It suffices to show that if

$$
\mu_{0} B_{\alpha}+\mu_{1} B_{\alpha-1}+\cdots+\mu_{2 m} B_{\alpha-2 m}=0,
$$

then $\mu_{0}=\mu_{1}=\cdots=\mu_{2 m}=0$. Indeed, by the definition of $B_{\alpha}$ in (3.1) and the unitary invariance of $d m$, we have that

$$
\begin{aligned}
0 & =\mu_{0} B_{\alpha} f(0)+\mu_{1} B_{\alpha-1} f(0)+\cdots+\mu_{2 m} B_{\alpha-2 m} f(0) \\
& =\int_{\mathbb{B}^{d}} f(w)\left(\mu_{0} c_{0}\left(1-|w|^{2}\right)^{\alpha}+\cdots+\mu_{2 m} c_{2 m}\left(1-|w|^{2}\right)^{\alpha-2 m}\right) d m(w)
\end{aligned}
$$

for any $f \in C\left(\overline{\mathbb{B}^{d}}\right)$. This implies that

$$
\mu_{0} c_{0}\left(1-|w|^{2}\right)^{\alpha}+\cdots+\mu_{2 m} c_{2 m}\left(1-|w|^{2}\right)^{\alpha-2 m} \equiv 0, w \in \mathbb{B}^{d},
$$

by the Riesz's representation theorem. Thus

$$
\mu_{0}=\mu_{1}=\cdots=\mu_{2 m}=0 .
$$

The following shows a direct connection between the Berezin transform $B_{\alpha, m}$ on $C_{\alpha, m}$ and the Berezin transform $B_{\alpha}$ on the standard weighted Bergman spaces.

Proposition 3.4. The Berezin transform $B_{\alpha, m}$ on $L^{1}\left(d \mu_{\alpha-2 m}\right)$ is a Berezin transform sum operator.

Proof. From the definition of $B_{\alpha, m}$ and Eq. (3.3), there are some real constants $\widetilde{c}(\alpha, j)$ such that for $f \in L^{1}\left(d \mu_{\alpha-2 m}\right)$ and $z \in \mathbb{B}^{d}$,

$$
\begin{aligned}
B_{\alpha, m} f(z) & \\
& =\int_{\mathbb{B}^{d}} f(w)\left|\mathbf{k}_{(\alpha, m)}(w, z)\right|^{2} d \mu_{\alpha}(w) \\
& =\int_{\mathbb{B}^{d}} f(w) \frac{\left(1-|z|^{2}\right)^{\alpha+d+1}}{\sum_{l=0}^{m} C_{l}|1-\langle z, w\rangle|^{2(\alpha+d+1)}} \sum_{j=0}^{2 m} \widetilde{c}(\alpha, j) \frac{|1-\langle z, w\rangle|^{2 j}}{\left(1-|z|^{2}\right)^{j}\left(1-|w|^{2}\right)^{j}} d \mu_{\alpha} \\
& =\sum_{j=0}^{2 m} \frac{\widetilde{c}(\alpha, j)}{\sum_{l=0}^{m} C_{l}} \int_{\mathbb{B}^{d}} f(w) \frac{\left(1-|z|^{2}\right)^{\alpha-j+d+1}}{|1-<z, w>|^{2(\alpha-j+d+1)}} d \mu_{\alpha-j}(w) \\
& =\sum_{j=0}^{2 m} \mu(\alpha, j) B_{\alpha-j} f(z),
\end{aligned}
$$


where $\mu(\alpha, j)=\frac{\widetilde{c}(\alpha, j)}{c_{\alpha-j} \sum_{l=0}^{m} C_{l}}$. It is easy to check that

$$
\mu(\alpha, 0)=\frac{\widetilde{c}(\alpha, 0)}{c_{\alpha} \sum_{l=0}^{m} C_{l}} \neq 0, \quad \mu(\alpha, 2 m)=\frac{\widetilde{c}(\alpha, 2 m)}{c_{\alpha-2 m} \sum_{l=0}^{m} C_{l}} \neq 0 .
$$

On the other hand, note that

$$
B_{\alpha, m} 1=1 \quad \text { and } \quad B_{\alpha-j} 1=1, j=0, \ldots, 2 m .
$$

It follows that $\sum_{j=0}^{2 m} \mu(\alpha, j)=1$, which leads to the desried result.

We remark some examples as follows.

Example. (1) When $m=0, B_{\alpha, 0}=B_{\alpha}$ is just the usual Berezin tansform.

(2) When $m=1$ and $d=1$, a direct computation shows

$$
B_{\alpha, 1}=\alpha^{2} B_{\alpha}-2\left(\alpha^{2}-1\right) B_{\alpha-1}+\left(\alpha^{2}-1\right) B_{\alpha-2} .
$$

Note that the second coefficient is not nonnegative even in the simplest case.

In the following, we will show some properties for the Berezin transform sum operator for the further research.

Lemma 3.5. Suppose $A$ is a Berezin transform sum operator. Then, for any $\varphi \in \operatorname{Aut}\left(\mathbb{B}^{d}\right)$,

$$
A(f \circ \varphi=A f) \circ \varphi
$$

Proof. It follows from Proposition 2.1 of [1] and Definition 3.3.

Proposition 3.6. Suppose $A$ is a Berezin transform sum operator. Then for $f \in C\left(\overline{\mathbb{B}^{d}}\right)$, we have that $A f \in C\left(\overline{\mathbb{B}^{d}}\right)$, and $A f=f$ on the boundary $\partial \mathbb{B}^{d}$.

Proof. By dominated convergence theorem, it can be shown that for each $\beta>-1, B_{\beta} f \in C\left(\overline{\mathbb{B}^{d}}\right)$ and $B_{\beta} f=f$ on the boundary $\partial \mathbb{B}^{d}$. See Proposition 6.14 in [21] for case $d=1$. Therefore, for a Berezin transform sum operator $A=\sum_{j=0}^{2 m} \mu_{j} B_{\alpha-j}$, one sees that $A f=\sum_{j=0}^{2 m} \mu_{j} B_{\alpha-j} f$ is smooth in the closed unit ball. For $z \in \partial \mathbb{B}^{d}$, we also have that

$$
A f(z)=\sum_{j=0}^{2 m} \mu_{j} B_{\alpha-j} f(z)=f(z) \sum_{j=0}^{2 m} \mu_{j}=f(z) .
$$

For $f \in C^{2}\left(\mathbb{B}^{d}\right)$, the invariant Laplacian $\widetilde{\Delta}$ is defined by

$$
\widetilde{\Delta} f(z)=\Delta\left(f \circ \varphi_{z}\right)(0), \quad \forall z \in \mathbb{B}^{d} .
$$

We say $f$ is $\mathcal{M}$-harmonic function if $\widetilde{\Delta} f=0$.

Proposition 3.7. Let $A$ be a Berezin transform sum operator. For $f \in L^{1}\left(\mathbb{B}^{d}\right.$, $\left.d \mu_{\alpha-2 m}\right) \cap C^{2}\left(\mathbb{B}^{d}\right)$, then $A f=f$ if $f$ is $\mathcal{M}$-harmonic. 
Proof. Since $f$ is $\mathcal{M}$-harmonic function, by Eq. (6) in [4] we know that

$$
B_{\alpha-j} f=f, \quad j=0, \ldots, 2 m .
$$

Write $A=\sum_{j=0}^{2 m} \mu_{j} B_{\alpha-j} \sum_{j=0}^{2 m} \mu_{j}=1$. Then

$$
A f=\sum_{j=0}^{2 m} \mu_{j} B_{\alpha-j} f=f .
$$

The converse of the above result is one of the center problem in the study of the Berezin transforms. In the case $B_{\alpha, m}$ over higher Cauchy-Riemann spaces, we establish the following result.

Theorem 3.8. Suppose $f \in C\left(\overline{\mathbb{B}^{d}}\right)$, then $B_{\alpha, m} f=f$ if and only if $f$ is $\mathcal{M}$ harmonic.

Proof. First we assume that $f$ is $\mathcal{M}$-harmonic. According to the above Proposition 3.7, we have that $B_{\alpha, m} f=f$.

Conversely, suppose that $B_{\alpha, m} f=f$. Let $h$ be the Poisson transform of the restriction of $f$ to the boundary $\partial \mathbb{B}^{d}$. Then $h$ is $\mathcal{M}$-harmonic function. Put $g=f-h$, then $g \in C_{0}\left(\overline{\mathbb{B}^{d}}\right)$ and $B_{\alpha, m} g=g$. We need to prove that $g$ is zero. Without loss of generality, we may assume that $g$ is a real function. Then it can get some extreme value inside $\mathbb{B}^{d}$. So, we may assume that there exists a point $a \in \mathbb{B}^{d}$ such that $g(z) \leq g(a)$ on $\mathbb{B}^{d}$. Then Lemma 3.5 and the definition of $B_{\alpha, m}$ implies that

$$
\begin{aligned}
0 & =B_{\alpha, m}(g(a)-g(z))(a) \\
& =B_{\alpha, m}\left(g \circ \varphi_{a}(0)-g \circ \varphi_{a}(w)\right)(0) \\
& =\int\left(g \circ \varphi_{a}(0)-g \circ \varphi_{a}(w)\right)\left|\mathbf{k}_{(\alpha, m), 0}(w)\right|^{2} d \mu_{\alpha} .
\end{aligned}
$$

Notice that $g \circ \varphi_{a}(0)-g \circ \varphi_{a}(w) \geq 0, \quad \forall w \in \mathbb{B}^{d}$. Then (3.6) means that

$$
\left(g \circ \varphi_{a}(0)-g \circ \varphi_{a}(w)\right) \mathbf{k}_{(\alpha, m), 0}(w)=0, \quad \forall w \in \mathbb{B}^{d} .
$$

Moreover, from (2.4), (2.6) and (3.2), we know that

$$
\begin{aligned}
\mathbf{k}_{(\alpha, m), 0}(w) & =\frac{\mathbf{K}_{(\alpha, m), 0}(w)}{\sqrt{\mathbf{K}_{(\alpha, m), 0}(0)}} \\
& =\frac{1}{\sum_{j=0}^{m} C_{j}} \sum_{j=0}^{m} C_{j} \sum_{k=0}^{j} \frac{(-j)_{k}(j-\alpha-1)_{k}}{k ! d !}\left(1-\frac{1}{1-|w|^{2}}\right)^{k} .
\end{aligned}
$$

By fundamental theorem of algebra, we know that the zero set of $\mathbf{k}_{(\alpha, m), 0}(w)$ on $\mathbb{B}^{d}$ is the union of at most finite spheres in the ball $\mathbb{B}^{d}$. This implies that

$$
g \circ \varphi_{a}(0)-g \circ \varphi_{a}(w)=0, \quad \forall w \in \mathbb{B}^{d} .
$$

Let $w=\varphi_{a}(z), z \in \mathbb{B}^{d}$ in (3.7), we get that

$$
g(z)=g(a), \quad \forall z \in \mathbb{B}^{d} .
$$

Since $g \in C_{0}\left(\overline{\mathbb{B}^{d}}\right)$ one sees that $g=0$, completing the proof. 
Corollary 3.9. Suppose $f \in C_{0}\left(\mathbb{B}^{d}\right)$, then $B_{\alpha, m} f=f$ if and only if $f=0$.

In the paper [10], Lee proved a fixed theorem in the case $f \in L^{\infty}\left(\mathbb{B}^{d}\right)$ when all the coefficients of the Berezin transform sum operator are nonnegative. In our situation, it would be hard to establish the fixed point theorem in the case $f \in L^{\infty}\left(\mathbb{B}^{d}\right)$.

\section{4. (Semi-)Commuting Toeplitz Operators on $C_{\alpha, m}$}

This section is devoted to the study for (semi-)commuting Toeplitz operators with bounded pluriharmonic symbols on $C_{\alpha, m}$. A function $f$ from $\mathbb{B}^{d}$ to $\mathbb{C}$ is said to be pluriharmonic, if $f$ is twice differentiable on $\mathbb{B}^{d}$ such that $f$ is harmonic on every complex line inside $\mathbb{B}^{d}$. It is equivalent to that there are two holomorphic functions $f_{1}$ and $f_{2}$ on $\mathbb{B}^{d}$, such that $f=f_{1}+\bar{f}_{2}$; see $[14,19]$. We first prove the following theorem for semi-commuting Toeplitz operators.

Theorem 4.1. Suppose that $f, g$ are two bounded pluriharmonic function on $\mathbb{B}^{d}$. Then $T_{f} T_{g}=T_{f g}$ on $C_{\alpha, m}$ if and only if $\bar{f}$ or $g$ is holomorphic.

The proof of the theorem are divided to several steps. Let $\mathcal{R}$ denote the radialization operator for continuous functions on $\mathbb{B}^{d}$, i.e. for any $f \in C\left(\mathbb{B}^{d}\right)$,

$$
\mathcal{R}(f)(w)=\int_{S} f(w \xi) d \sigma(\xi), \quad w \in \mathbb{B}^{d} .
$$

One important property for $\mathcal{R}$ is that it commutes with usual weighted Berezin transform and also commutes with the invariant Laplacian $\widetilde{\Delta}$.

Lemma 4.2. (1) If $f \in L^{1}\left(d \mu_{\beta}\right)$ is continuous, then $\mathcal{R}\left(B_{\beta} f\right)=B_{\beta}(\mathcal{R} f)$.

(2) If $f$ is twice differentiable on $\mathbb{B}^{d}$, then $\widetilde{\Delta}(\mathcal{R} f)=\mathcal{R}(\widetilde{\Delta} f)$.

Proof. (1) It comes from Fubini's Theorem and unitary invariance of $d \mu_{\beta}$.

(2) It follows from Theorem 4.1.3 of [14] and differentiation under integral sign. See Lemma 6.27 in [21] for case $d=1$.

We next show a fixed point theorem for the general functions.

Proposition 4.3. Suppose $f \in L^{1}\left(d \mu_{\alpha-2 m}\right)$, then $f$ is $\mathcal{M}$-harmonic if and only if $B_{\alpha, m} f=f$ and $\mathcal{R}(f \circ \varphi) \in C\left(\overline{\mathbb{B}^{d}}\right)$ for any $\varphi \in \operatorname{Aut}\left(\mathbb{B}^{d}\right)$.

Proof. First we assume that $f$ is $\mathcal{M}$-harmonic function. Then by Proposition 3.7 , we have that $B_{\alpha, m} f=f$. By $\mathcal{M}$-mean value property of $\mathcal{M}$-harmonic functions [14], one sees

$$
\mathcal{R}(f \circ \varphi)(w)=f \circ \varphi(0)
$$

is a constant function.

Conversely, assume that $B_{\alpha, m} f=f$, and $\mathcal{R}(f \circ \varphi) \in C\left(\overline{\mathbb{B}^{d}}\right), \quad \forall \varphi \in$ Aut $\left(\mathbb{B}^{d}\right)$. Then Lemma 3.5 implies that

$$
B_{\alpha, m}(f \circ \varphi)=f \circ \varphi \text {. }
$$

Together with (1) of Lemma 4.2, one sees that

$$
B_{\alpha, m}(\mathcal{R}(f \circ \varphi))=\mathcal{R}(f \circ \varphi) .
$$


From Theorem 3.8, this implies that $\mathcal{R}(f \circ \varphi)$ is $\mathcal{M}$-harmonic. Note that any radializational $\mathcal{M}$-harmonic is constant [14]. Therefore, for any $\varphi \in \operatorname{Aut}\left(\mathbb{B}^{d}\right)$,

$$
f \circ \varphi(0)=\mathcal{R}(f \circ \varphi)(w)=\int_{S} f \circ \varphi(w \xi) d \sigma(\xi), \quad w \in \mathbb{B}^{d},
$$

Moreover, $f=B_{\alpha, m} f$ is smooth inside $\mathbb{B}^{d}$. By Corollary 2 of Theorem 4.2.4 of [14], we have that $f$ is $\mathcal{M}$-harmonic.

For $p \geq 1$, denote $H^{p}\left(\mathbb{B}^{d}\right)$ by the Hardy space on $\mathbb{B}^{d}$; see for example [14]. For bounded pluriharmonic functions, we have the following results.

Lemma 4.4. Suppose that $u=f+\bar{g}$ is bounded pluriharmonic function on $\mathbb{B}^{d}$, where $f, g$ are holomorphic functions. Then for any $\varphi \in A u t\left(\mathbb{B}^{d}\right)$,

(1) $f \circ \varphi, g \circ \varphi \in H^{p}\left(\mathbb{B}^{d}\right)$ for any $p>1$.

(2) $f, g \in L_{a}^{2}\left(d \mu_{\beta}\right), \quad \forall \beta>-1$.

(3) $\mathcal{R}((f \bar{g}) \circ \varphi) \in C\left(\overline{\mathbb{B}^{d}}\right)$.

Proof. (1) It follows from Proposition 10 of [19].

(2) From (1), we know that $f, g \in H^{2}\left(\mathbb{B}^{d}\right)$. By using the integration formula in polar coordinates, we get the desired result.

(3) To ease the notation, we denote $h=f \circ \varphi, l=g \circ \varphi$. From (1) one sees that $h, l \in H^{2}\left(\mathbb{B}^{d}\right)$. Let $h^{*}(\xi), l^{*}(\xi)$ be the $K$-limit of $h, l$ on $\partial \mathbb{B}^{d}$ respectively. By [14], we have that $f, g \in H^{2}\left(\mathbb{B}^{d}\right)$.

$$
\lim _{r \rightarrow 1^{-}} \int_{S} h(r \xi) \bar{l}(r \xi) d \sigma=\int_{S} h^{*}(\xi) \bar{l}^{*}(\xi) d \sigma .
$$

Therefore, $\mathcal{R}(h \bar{l}) \in C\left(\overline{\mathbb{B}^{d}}\right)$. This means that $\mathcal{R}((f \bar{g}) \circ \varphi) \in C\left(\overline{\mathbb{B}^{d}}\right)$, completing the proof.

Lemma 4.5. (1) The reproducing kernel $\boldsymbol{K}_{(\alpha, m)}(z, w)$ of $C_{\alpha, m}$ has the form

$$
\begin{aligned}
\boldsymbol{K}_{(\alpha, m)}(z, w)= & \sum_{|I|=m} \frac{\bar{z}^{I}}{\left(1-|z|^{2}\right)^{m}} \frac{P_{I}(z, w)}{(1-\langle z, w\rangle)^{\alpha+d+1-n_{I}\left(1-|w|^{2}\right)^{n_{I}}}} \\
& +\sum_{|I|=m-1} \frac{\bar{z}^{I}}{\left(1-|z|^{2}\right)^{m-1}} \frac{P_{I}(z, w)}{(1-\langle z, w\rangle)^{\alpha+d+1-n_{I}\left(1-|w|^{2}\right)^{n_{I}}}} \\
& +\cdots+\frac{P_{0}(z, w)}{(1-\langle z, w\rangle)^{\alpha+d+1}}
\end{aligned}
$$

$z, w \in \mathbb{B}^{d}$, where $P_{I}(z, w) \in \mathbb{C}[z, w], n_{I} \in\{0,1, \ldots, m\}$.

(2) Suppose that $f=f_{1}+\bar{f}_{2}$ is bounded pluriharmonic function on $\mathbb{B}^{d}$, where $f_{1}, f_{2}$ are holomorphic functions. Then the following equality holds

$$
T_{f} \boldsymbol{K}_{(\alpha, m), z}(w)=\left(f_{1}(w)+\bar{f}_{2}(z)\right) \boldsymbol{K}_{(\alpha, m), z}(w)
$$


Proof. (1) The reproducing kernel is given by formula (2.3). Then, by the binomial theorem we have

$$
\begin{aligned}
\mathbf{K}_{(\alpha, m)}(z, w) & \\
= & \frac{1}{(1-\langle z, w\rangle)^{\alpha+d+1}} \sum_{j=0}^{m} c(\alpha, j) \frac{|1-\langle z, w\rangle|^{2 j}}{\left(1-|z|^{2}\right)^{j}\left(1-|w|^{2}\right)^{j}} \\
& =\sum_{j=0}^{m} \frac{c(\alpha, j)}{(1-\langle z, w\rangle)^{\alpha+d+1-j}} \frac{1}{\left(1-|w|^{2}\right)^{j}} \frac{\sum_{k=0}^{j}(-1)^{k}\left(\begin{array}{l}
j \\
k
\end{array}\right) \sum_{|\beta|=k}\left(\begin{array}{c}
k \\
\beta
\end{array}\right) w^{\beta} \bar{z}^{\beta}}{\left(1-|z|^{2}\right)^{j}} \\
& =\sum_{j=0}^{m} \frac{c(\alpha, j)}{(1-\langle z, w\rangle)^{\alpha+d+1-j}\left(1-|w|^{2}\right)^{j}} \sum_{k=0}^{j}(-1)^{k}\left(\begin{array}{l}
j \\
k
\end{array}\right) \sum_{|\beta|=k}\left(\begin{array}{l}
k \\
\beta
\end{array}\right) w^{\beta} \bar{z}^{\beta} \frac{\left(1+\frac{|z|^{2}}{1-|z|^{2}}\right)^{j-k}}{\left(1-|z|^{2}\right)^{k}} \\
& =\sum_{j=0}^{m} \frac{c(\alpha, j)}{(1-\langle z, w\rangle)^{\alpha+d+1-j}\left(1-|w|^{2}\right)^{j}} \sum_{k=0}^{j}(-1)^{k}\left(\begin{array}{l}
j \\
k
\end{array}\right) \sum_{|\beta|=k}\left(\begin{array}{l}
k \\
\beta
\end{array}\right) w^{\beta} \bar{z}^{\beta} \sum_{n=0}^{j-k} \frac{|z|^{2 n}}{\left(1-|z|^{2}\right)^{n+k}} \\
& =\sum_{j=0}^{m} \frac{c(\alpha, j)}{(1-\langle z, w\rangle)^{\alpha+d+1-j}\left(1-|w|^{2}\right)^{j}} \sum_{k=0}^{j}(-1)^{k}\left(\begin{array}{c}
j \\
k
\end{array}\right) \sum_{|\beta|=k} \sum_{n=0}^{j-k} \sum_{|\gamma|=n} \frac{\left(\begin{array}{l}
k \\
\beta
\end{array}\right) \bar{z}^{\beta+\gamma} w^{\beta} z^{\gamma}}{\left(1-|z|^{2}\right)^{n+k}} .
\end{aligned}
$$

It leads to the desired result.

(2) From (2) of Lemma 4.4, we know that

$$
f_{1}, f_{2} \in L_{a}^{2}\left(d \mu_{\alpha-j}\right), j=0, \ldots, 2 m .
$$

Together with (1) and the definition of $C_{\alpha, m}$, it follows that

$$
f_{1} \mathbf{K}_{(\alpha, m), z}, f_{2} \mathbf{K}_{(\alpha, m), z} \in C_{\alpha, m} .
$$

Thus we have

$$
\begin{aligned}
T_{f} \mathbf{K}_{(\alpha, m), z}(w) & =\left\langle\left(f_{1}+\bar{f}_{2}\right) \mathbf{K}_{(\alpha, m), z}, \mathbf{K}_{(\alpha, m), w}\right\rangle \\
& =\left\langle f_{1} \mathbf{K}_{(\alpha, m), z}, \mathbf{K}_{(\alpha, m), w}\right\rangle+\left\langle\mathbf{K}_{(\alpha, m), z}, f_{2} \mathbf{K}_{(\alpha, m), w}\right\rangle \\
& =\left(f_{1}(w)+\bar{f}_{2}(z)\right) \mathbf{K}_{(\alpha, m), z}(w) .
\end{aligned}
$$

We also need the following two results from [20].

Theorem 4.6. [20] Suppose that $f, g, h, l \in H^{2 d}\left(\mathbb{B}^{d}\right)$. Then $f \bar{g}-h \bar{l}$ is $\mathcal{M}$ harmonic if and only if one of the following conditions holds:

(a) Both $f$ and $h$ are constants.

(b) Both $f$ and $l$ are constants.

(c) Both $g$ and $h$ are constants.

(d) Both $g$ and $l$ are constants.

(e) There is a nonzero constant $b$ such that $b g-l$ and $f-\bar{b} h$ are constants.

Proof. See Theorem 5.6 of [20].

Corollary 4.7. $[19,20]$ Suppose that $f, g \in H^{2 d}\left(\mathbb{B}^{d}\right)$. Then $f \bar{g}$ is $\mathcal{M}$-harmonic if and only if $f$ or $g$ is constant.

Now we turn to the proof of Theorem 4.1.

The Proof of Theorem 4.1. Note that $C_{\alpha, m}$ is invariant under the multiplication by bounded holomorphic functions. It is trivial that $T_{f} T_{g}=T_{f g}$ when $\bar{f}$ or $g$ is holomorphic. 
Conversely, suppose that $T_{f} T_{g}=T_{f g}$ on $C_{\alpha, m}$. Assume that $f=f_{1}+$ $\bar{f}_{2}, g=g_{1}+\bar{g}_{2}$, where $f_{1}, f_{2}, g_{1}, g_{2}$ are holomorphic.

By (2) of Lemma 4.5, it is clear that

$$
T_{\bar{f}} \mathbf{k}_{(\alpha, m), z}(w)=\left(f_{2}(w)+\bar{f}_{1}(z)\right) \mathbf{k}_{(\alpha, m), z}(w),
$$

and

$$
T_{g} \mathbf{k}_{(\alpha, m), z}(w)=\left(g_{1}(w)+\bar{g}_{2}(z)\right) \mathbf{k}_{(\alpha, m), z}(w) .
$$

According to (1) and (2) of Lemma 4.3, we know that

$$
f_{1} g_{1}, f_{2} g_{2} \in L_{a}^{2}\left(d \mu_{\alpha-2 m}\right)
$$

and

$$
f_{1} \bar{g}_{2}, \bar{f}_{2} g_{1} \in L^{1}\left(d \mu_{\alpha-2 m}\right) .
$$

Then, by (4.1)-(4.4), we have that

$$
\begin{aligned}
0 & =B_{\alpha, m}\left(T_{f g}-T_{f} T_{g}\right)(z) \\
& =B_{\alpha, m}(f g)-\left\langle\left(g_{1}+\bar{g}_{2}(z)\right) \mathbf{k}_{(\alpha, m), z},\left(\bar{f}_{1}(z)+f_{2}\right) \mathbf{k}_{(\alpha, m), z}\right\rangle \\
& =B_{\alpha, m}\left(f_{1} \bar{g}_{2}\right)(z)-f_{1}(z) \bar{g}_{2}(z), \quad z \in \mathbb{B}^{d} .
\end{aligned}
$$

That is, $B_{\alpha, m}\left(f_{1} \bar{g}_{2}\right)=f_{1} \bar{g}_{2}$. Moreover, from (4.4) and the proof of (3) of Lemma 4.4, we know that

$$
\mathcal{R}\left(\left(f_{1} \bar{g}_{2}\right) \circ \varphi\right) \in C\left(\overline{\mathbb{B}^{d}}\right), \quad \forall \varphi \in \operatorname{Aut}\left(\mathbb{B}^{d}\right) .
$$

Then $f_{1} \bar{g}_{2}$ is $\mathcal{M}$-harmonic by Proposition 4.3. According to (1) of Lemma 4.4 and Corollary 4.7, we know that $f_{1}$ or $g_{2}$ is constant. Thus $\bar{f}$ or $g$ is bounded holomorphic.

In the rest of this section, we prove the following theorem for commuting Toeplitz operators.

Theorem 4.8. Suppose that $f, g$ are two bounded pluriharmonic function on $\mathbb{B}^{d}$. Then $T_{f} T_{g}=T_{g} T_{f}$ on $C_{\alpha, m}$ if and only if $f$ and $g$ satisfy one of the following conditions:

(1) Both $f$ and $g$ are holomorphic.

(2) Both $\bar{f}$ and $\bar{g}$ are holomorphic.

(3) Either $f$ or $g$ is constant on $\mathbb{B}^{d}$.

(4) There is a nonzero constant b such that $f-b g$ is constant on $\mathbb{B}^{d}$.

Proof. It is easy to check that

$$
T_{f} T_{g}=T_{g} T_{f}
$$

if $f$ and $g$ satisfy one of conditions (1)-(4).

In the next, we will prove another direction. Suppose $f=f_{1}+\bar{f}_{2}, g=$ $g_{1}+\bar{g}_{2}$ are two bounded pluriharmonic function on $\mathbb{B}^{d}$ with $f_{1}, f_{2}, g_{1}, g_{2}$ holomorphic functions on $\mathbb{B}^{d}$ and $T_{f} T_{g}=T_{g} T_{f}$ on $C_{\alpha, m}$. According to (4.1)(4.4), we know that

$$
B_{\alpha, m}\left(T_{f} T_{g}\right)=B_{\alpha, m}\left(g_{1} \bar{f}_{2}\right)+f_{1} g_{1}+\bar{f}_{2} \bar{g}_{2}+f_{1} \bar{g}_{2},
$$

and

$$
B_{\alpha, m}\left(T_{g} T_{f}\right)=B_{\alpha, m}\left(f_{1} \bar{g}_{2}\right)+g_{1} f_{1}+\bar{g}_{2} \bar{f}_{2}+g_{1} \bar{f}_{2}
$$


Using (4.5)-(4.6), one sees that

$$
B_{\alpha, m}\left(g_{1} \bar{f}_{2}-f_{1} \bar{g}_{2}\right)=g_{1} \bar{f}_{2}-f_{1} \bar{g}_{2} .
$$

From (4.4) and the proof of (3) of Lemma 4.4, we know that

$$
\mathcal{R}\left(\left(g_{1} \bar{f}_{2}-f_{1} \bar{g}_{2}\right) \circ \varphi\right) \in C\left(\overline{\mathbb{B}^{d}}\right), \quad \forall \varphi \in A u t\left(\mathbb{B}^{d}\right) .
$$

Then $g_{1} \bar{f}_{2}-f_{1} \bar{g}_{2}$ is $\mathcal{M}$-harmonic by Proposition 4.2 . According to (1) of Lemma 4.4 and Theorem 4.6, we have that $f_{1}, f_{2}, g_{1}, g_{2}$ satisfy one of the following conditions:

(a) Both $g_{1}$ and $f_{1}$ are constants.

(b) Both $g_{1}$ and $g_{2}$ are constants.

(c) Both $f_{2}$ and $f_{1}$ are constants.

(d) Both $f_{2}$ and $g_{2}$ are constants.

(e) There is a nonzero constant $b$ such that $b f_{2}-g_{2}$ and $g_{1}-\bar{b} f_{1}$ are constants.

It is easy to see that (a) implies (2), (b) implies (1), (c) implies (3), (d) implies (3) and (e) implies (4).

\section{Compact Toeplitz Operators on $C_{\alpha, m}$}

In this section we discuss compact products of finite Toeplitz operators with bounded pluriharmonic symbol functions on $C_{\alpha, m}$. We first show the following theorem for compact single Toeplitz operator.

Theorem 5.1. Let $f$ be bounded pluriharmonic function on $\mathbb{B}^{d}$. Then $T_{f}$ is compact on $C_{\alpha, m}$ if and only if $f=0$.

To complete the proof, we need two lemmas.

Lemma 5.2. (1) $\boldsymbol{k}_{(\alpha, m), z} \rightarrow 0$ weakly on $C_{\alpha, m}$ as $z \rightarrow \partial \mathbb{B}^{d}$.

(2) $k_{\alpha, z} \rightarrow 0$ weakly on $C_{\alpha, m}$ as $z \rightarrow \partial \mathbb{B}^{d}$.

Proof. (1) From (2.2), the set $\left\{f=\sum_{j=0}^{m} \sum_{|I|=j} \frac{\bar{z}^{I}}{\left(1-|z|^{2}\right)^{m}} p_{I}: p_{I} \in \mathbb{C}[z], \quad \forall I\right\}$ is dense in $C_{\alpha, m}$. So it suffices to show that $\left\langle\mathbf{k}_{(\alpha, m), z}(w), \frac{\bar{w}^{L}}{\left(1-|w|^{2}\right)^{|L|}} p(w)\right\rangle \rightarrow 0$ as $z \rightarrow \partial \mathbb{B}^{d}$, for any holomorphic polynomial $p$ and multi-index $L$ such that $0 \leq|L| \leq m \leq D(\alpha)$. Indeed, for any $z \in \mathbb{B}^{d}$, we have

$$
\begin{aligned}
& \left|\left\langle\mathbf{k}_{(\alpha, m), z}(w), \frac{\bar{w}^{L}}{\left(1-\left.|w|^{2}\right|^{|L|}\right.} p(w)\right\rangle\right| \\
& \quad=\left|\left\langle\frac{1}{\sqrt{\sum C_{n}}} k_{\alpha, z}(w) \sum_{j=0}^{m} c(\alpha, j) \frac{|1-<w, z>|^{2 j}}{\left(1-|w|^{2}\right)^{j}\left(1-|z|^{2}\right)^{j}}, \frac{\bar{w}^{L}}{\left(1-|w|^{2}\right)^{|L|}} p(w)\right\rangle\right| \\
& \quad \leq \sum_{j=0}^{m} \frac{|c(\alpha, j)|}{\sqrt{\sum C_{n}}}\left(1-|z|^{2}\right)^{\frac{\alpha-2 j+d+1}{2}}\|p\|_{\infty} \int_{\mathbb{B}^{d}} \frac{\left(1-|w|^{2}\right)^{\alpha-|L|-j}}{|1-\langle w, z\rangle|^{d+1+\alpha-2 j}} d m(w) .
\end{aligned}
$$


Set $J_{\alpha,|L|, j}(z)=\int_{\mathbb{B}^{d}} \frac{\left(1-|w|^{2}\right)^{\alpha-|L|-j}}{|1-<w, z>|^{d+1+\alpha-|L|-j+|L|-j}} d v(w), \quad \forall z \in \mathbb{B}^{d}$. Note that $0 \leq|L|, j \leq D(\alpha)<\frac{\alpha+1}{2}$, so $\alpha-|L|-j>-1$. Then, by Proposition 1.4.10 of [14], we get that

$$
J_{\alpha,|L|, j}(z) \sim\left\{\begin{array}{l}
1,|L|-j<0 \\
\log \frac{1}{1-|z|^{2}},|L|-j=0 ; \\
\frac{1}{\left(1-|z|^{2}\right)^{l-j}},|L|-j>0 .
\end{array} \quad z \rightarrow \partial \mathbb{B}^{d}\right.
$$

Since $0 \leq j \leq m<\frac{\alpha+1}{2}$ and $0 \leq|L| \leq m<\frac{\alpha+1}{2}$, it follows that

$$
\left(1-|z|^{2}\right)^{\frac{\alpha-2 j+d+1}{2}} J_{\alpha,|L|, j}(z) \rightarrow 0, z \rightarrow \partial \mathbb{B}^{d} .
$$

Thus, by (5.1) we get that

$$
\left\langle\mathbf{k}_{(\alpha, m), z}(w), \frac{\bar{w}^{L}}{\left(1-|w|^{2}\right)^{|L|}} p(w)\right\rangle \rightarrow 0, z \rightarrow \partial \mathbb{B}^{d} .
$$

This completes the proof.

(2) The proof is similar to (1). We omit it.

Lemma 5.3. Let $T_{f}$ be compact Toeplitz operator on $C_{\alpha, m}$ with symbol $f \in$ $L^{\infty}\left(\mathbb{B}^{d}\right)$. Then $B_{\alpha, m} f(z) \rightarrow 0, z \rightarrow \partial \mathbb{B}^{d}$.

Proof. Since $T_{f}$ is compact Toeplitz operator on $C_{\alpha, m}$ with bounded symbol $f$, it follows that

$$
T_{f} \mathbf{k}_{(\alpha, m), z} \stackrel{\|\cdot\|}{\longrightarrow} 0, \quad z \rightarrow \partial \mathbb{B}^{d},
$$

by (1) of Lemma 5.1. From the definition of Berezin transform and Cauchy's inequality, we have that

$$
\left|B_{\alpha, m} f(z)\right|=\mid\left\langle T_{f} \mathbf{k}_{(\alpha, m), z}, \mathbf{k}_{(\alpha, m), z}\right\rangle \leq\left\|T_{f} \mathbf{k}_{(\alpha, m), z}\right\| \rightarrow 0, \quad z \rightarrow \partial \mathbb{B}^{d} .
$$

When $m=0$, i.e. in the case weighted Bergman space $L_{a}^{2}\left(d \mu_{\alpha}\right)$, we know that the converse of Lemma 5.3 is also true, and it is even true in the weighted Bergman spaces over bounded symmetric domains [5]. However, it is unknown how to characterize the compact Toeplitz operators with bounded symbols on $C_{\alpha, m}$ for $m>0$.

Now we prove the Theorem 5.1.

The Proof of Theorem 5.1. Suppose $f=0$, then $T_{f}=0$ is compact on $C_{\alpha, m}$.

Conversely, suppose $f$ is bounded pluriharmonic function. Note that pluriharmonic functions are $\mathcal{M}$-harmonic functions. Then by Proposition 3.7, it follows that

$$
B_{\alpha, m} f(z)=f(z) .
$$

Hence, together with Lemma 5.3, we get that

$$
f(z)=B_{\alpha, m} f(z) \rightarrow 0, \quad z \rightarrow \partial \mathbb{B}^{d} .
$$

Note that $f$ is pluriharmonic on $\mathbb{B}^{d}$, then $f=0$ on $\mathbb{B}^{d}$, by Theorem 4.3.3 of [14].

In the rest of the section, we consider the problem of compact finite products of Toeplitz operators. Inspired by [3] we show the following theorem. 
Theorem 5.4. $(d \geq 2)$ Let $u_{1}, \ldots, u_{n}$ be bounded pluriharmonic functions on $\mathbb{B}^{d}$, and suppose that $u_{1}, \ldots, u_{n} \in C\left(\mathbb{B}^{d} \cup W\right)$ for some nonempty relatively open set $W \subseteq \partial \mathbb{B}^{d}$. Then $\prod_{j=1}^{n} T_{u_{j}}$ is compact on $C_{\alpha, m}$ if and only if $u_{j}=0$ on $\mathbb{B}^{d}$ for some $j$.

As a consequence, we immediately obtain the following result about zero product problems of the Toeplitz operators on $C_{\alpha, m}$.

Corollary 5.5. $(d \geq 2)$ Let $u_{1}, \ldots, u_{n}$ be bounded pluriharmonic functions on $\mathbb{B}^{d}$, and suppose that $u_{1}, \ldots, u_{n} \in C\left(\mathbb{B}^{d} \cup W\right)$ for some nonempty relatively open set $W \subseteq \partial \mathbb{B}^{d}$. Then $\prod_{j=1}^{n} T_{u_{j}}=0$ on $C_{\alpha, m}$ if and only if $u_{j}=0$ on $\mathbb{B}^{d}$ for some $j$.

The proof of Theorem 5.4 is divided several steps. Recall firstly the unitary representation $U$ of $A u t\left(\mathbb{B}^{d}\right)$ on $L^{2}\left(d \mu_{\alpha}\right)$ as mentioned in Sect. 2.

Proposition 5.6. Suppose that $f \in L^{\infty}\left(\mathbb{B}^{d}\right)$ and $\varphi \in A u t\left(\mathbb{B}^{d}\right)$, then

$$
U_{\varphi} T_{f} U_{\varphi^{-1}}=T_{f \circ \varphi}
$$

Proof. Since $U_{\varphi}$ maps $C_{\alpha, m}$ onto $C_{\alpha, m}$ and $U_{\varphi}$ is unitary, it follows that

$$
P U_{\varphi}=U_{\varphi} P
$$

where $P$ is the orthogonal projection from $L^{2}\left(d \mu_{\alpha}\right)$ onto $C_{\alpha, m}$. Then, $\forall g \in$ $C_{\alpha, m}$, we have that

$$
T_{f \circ \varphi} U_{\varphi} g=P_{m}\left(f \circ \varphi \cdot g \circ \varphi\left(J_{\varphi} z\right)^{\frac{\alpha+d+1}{d+1}}\right)=U_{\varphi} T_{f} g
$$

Corollary 5.7. $U_{\varphi_{a}} T_{f} U_{\varphi_{a}}=T_{f \circ \varphi_{a}}, \quad \forall a \in \mathbb{B}^{d}$.

To prove Theorem 5.4, we give the $\alpha$-Berezin transform on $C_{\alpha, m}$. The $\alpha$-Berezin transform of $T \in B\left(C_{\alpha, m}\right)$ is defined by

$$
B_{\alpha}(T)(z)=\left\langle T k_{\alpha, z}, k_{\alpha, z}\right\rangle, \quad z \in \mathbb{B}^{d},
$$

where $k_{\alpha, z}(w)$ is the normalized reproducing kernel of Bergman space $L_{a}^{2}\left(d \mu_{\alpha}\right)$. For operator $T$ that can be written the finite sum of products of finite Toeplitz operators, we have the following result.

Proposition 5.8. Suppose $T=\sum_{j=1}^{l} \prod_{k=1}^{n_{j}} T_{u_{j, k}}$, if each function $u_{j, k} \in$ $L^{\infty}\left(\mathbb{B}^{d}\right) \cap C\left(\mathbb{B}^{d} \cup\{\xi\}\right)$, where $\xi \in \mathbb{B}^{d}$. Then $B_{\alpha}(T) \in C\left(\mathbb{B}^{d} \cup\{\xi\}\right)$ and $B_{\alpha}(T)(\xi)=\sum_{j=1}^{l} \prod_{k=1}^{n_{j}} u_{j, k}(\xi)$.

Proof. In fact, it suffices to show the formula when $T=\prod_{j=1}^{n} T_{u_{j}}$. Note that $U_{\varphi_{z}} 1=k_{\alpha, z}, \quad \forall z \in \mathbb{B}^{d}$. Together with Corollary 5.7, it follows that

$$
B_{\alpha}(T)(z)=\left\langle\prod_{j=1}^{n} T_{u_{j}} k_{\alpha, z}, k_{\alpha, z}\right\rangle=\left\langle\prod_{j=1}^{n} T_{u_{j} \circ \varphi_{z}} 1,1\right\rangle .
$$


Then, by Cauchy's inequality, it implies that

$$
\begin{aligned}
\left|B_{\alpha}(T)(z)-\prod_{j=1}^{n} u_{j}(\xi)\right| & =\left|\left\langle\prod_{j=1}^{n} T_{u_{j} \circ \varphi_{z}} 1-\prod_{j=1}^{n} u_{j}(\xi), 1\right\rangle\right| \\
& \leq\left\|\prod_{j=1}^{n} T_{u_{j} \circ \varphi_{z}} 1-\prod_{j=1}^{n} u_{j}(\xi)\right\|
\end{aligned}
$$

Thus the proof will be completed by (5.3) if we prove that

$$
\left\|\prod_{j=1}^{n} T_{u_{j} \circ \varphi_{z}} 1-\prod_{j=1}^{n} u_{j}(\xi)\right\| \rightarrow 0, \quad z \rightarrow \xi
$$

In the next, we will prove (5.4) by induction. For $n=1$. We know that function $\varphi_{z} \rightarrow \xi$ pointwisely as $z \rightarrow \xi$. Since $u_{1} \in L^{\infty}\left(\mathbb{B}^{d}\right) \cap C\left(\mathbb{B}^{d} \cup\{\xi\}\right)$, it implies that

$$
\left\|u_{1} \circ \varphi_{z}-u_{1}(\xi)\right\| \rightarrow 0, z \rightarrow \xi,
$$

by the dominated convergence theorem. Then

$\left\|T_{u_{1} \circ \varphi_{z}} 1-u_{1}(\xi)\right\|=\left\|P\left(u_{1} \circ \varphi_{z}-u_{1}(\xi)\right)\right\| \leq\left\|u_{1} \circ \varphi_{z}-u_{1}(\xi)\right\| \rightarrow 0, \quad z \rightarrow \xi$,

where $P$ is the orthogonal projection from $L^{2}\left(d \mu_{\alpha}\right)$ onto $C_{\alpha, m}$. This proves (5.4) for $n=1$. We now proceed by induction on $n$. Assume that (5.4) holds for some $n \geq 1$ and consider the case $n+1$. By induction hypothesis, we have that

$$
\begin{aligned}
& \left\|\prod_{j=1}^{n+1} T_{u_{j} \circ \varphi_{z}} 1-\prod_{j=1}^{n+1} u_{j}(\xi)\right\| \\
& \quad \leq\left\|T_{u_{1} \circ \varphi_{z}}\right\| \cdot\left\|\prod_{j=2}^{n+1} T_{u_{j} \circ \varphi_{z}} 1-\prod_{j=2}^{n+1} u_{j}(\xi)\right\|+\left\|T_{u_{1} \circ \varphi_{z}} 1-u_{1}(\xi)\right\| \cdot\left\|\prod_{j=2}^{n+1} u_{j}\right\|_{\infty} \\
& \quad \rightarrow 0, \quad z \rightarrow \xi .
\end{aligned}
$$

It means that (5.4) holds for $n+1$. This completes the induction and the proof of the proposition.

The Proof of Theorem 5.4 Suppose $u_{j}=0$ on $\mathbb{B}^{d}$ for some $j$, then $\prod_{j=1}^{n} T_{u_{j}}=$ 0 is compact on $C_{\alpha, m}$.

Conversely, suppose $\prod_{j=1}^{n} T_{u_{j}}$ is compact on $C_{\alpha, m}$. By (2) of Lemma 5.2, we know that $k_{\alpha, z} \rightarrow 0$ weakly on $C_{\alpha, m}, z \rightarrow \partial \mathbb{B}^{d}$. Since $\prod_{j=1}^{n} T_{u_{j}}$ is compact on $C_{\alpha, m}$, it follows that $\prod_{j=1}^{n} T_{u_{j}} k_{\alpha, z}$ converges to 0 on norm topology as $z \rightarrow \partial \mathbb{B}^{d}$. Then, we have that

$$
\left|B_{\alpha}\left(\prod_{j=1}^{n} T_{u_{j}}\right)(z)\right|=\mid\left\langle\prod_{j=1}^{n} T_{u_{j}} k_{\alpha, z}, k_{\alpha, z}\right\rangle \leq\left\|\prod_{j=1}^{n} T_{u_{j}} k_{\alpha, z}\right\| \rightarrow 0, \quad z \rightarrow \partial \mathbb{B}^{d} .
$$

Since $u_{1}, \ldots, u_{n} \in C\left(\mathbb{B}^{d} \cup W\right)$ for some nonempty relatively open set $W \subseteq$ $\partial \mathbb{B}^{d}$, together with Proposition 5.8, we get that 


$$
\prod_{j=1}^{n} u_{j}(\xi)=0, \quad \forall \xi \in W .
$$

Then $u_{j}=0$ on $\mathbb{B}^{d}$ for some $j$, by Corollary 3.5 of [3].

\section{Acknowledgements}

The authors would like to express their great gratitude to Professor K. Guo for his valuable guidance and encouragement over the years. The authors would also like to thank Professor G. Zhang for his helpful discussions and support.

Open Access. This article is distributed under the terms of the Creative Commons Attribution 4.0 International License (http://creativecommons.org/licenses/by/4. $0 /$ ), which permits unrestricted use, distribution, and reproduction in any medium, provided you give appropriate credit to the original author(s) and the source, provide a link to the Creative Commons license, and indicate if changes were made.

\section{References}

[1] Ahern, P., Flores, M., Rudin, W.: An invariant volume-mean-value property. J. Funct. Anal. 111, 380-397 (1993)

[2] Brown, A., Halmos, P.: Algebraic properties of Toeplitz operators. J. Reine Angew. Math. 213, 89-102 (1964)

[3] Choe, B., Koo, H., Lee, J.: Toeplitz products with pluriharmonic symbols on the Hardy space over the ball. J. Math. Anal. Appl. 381, 365-382 (2011)

[4] Engliš, M.: Functions invariant under the Berezin transform. J. Funct. Anal. 121, 233-254 (1994)

[5] Engliš, M.: Compact Toeplitz operators via the Berezin transform on bounded symmetric domains. Integr. Equ. Oper. Theory 33, 426-455 (1999)

[6] Engliš, M., Peetre, J.: Covariant Cauchy-Riemann operators and higher Laplacians on Kähler manifolds. J. Reine Angew. Math. 478, 17-56 (1996)

[7] Engliš, M., Peetre, J.: Covariant differential operators and Green's functions. Ann. Polon. Math. 66, 77-103 (1997)

[8] Engliš, M., Zhang, G.: Toeplitz operators on higher Cauchy-Riemann spaces. Doc. Math. 22, 1081-1116 (2017)

[9] Faraut, J., Korányi, A.: Analysis on Symmetric Cones. The Clarendon Press, New York (1994)

[10] Lee, J.: Properties of the Berezin transform of bounded functions. Bull. Aust. Math. Soc. 59, 21-31 (1999)

[11] Loos, O.: Jordan Pairs, Lecture Notes in Mathematics, vol. 460. Springer, Berlin (1975)

[12] Peetre, J., Peng, L., Zhang, G.: A Weighted Plancherel formula. I: The Case of the Disk, Applications to Hankel Operators. Technical Report, Stockholm

[13] Peetre, J., Zhang, G.: Invariant Cauchy-Riemann operators and relative discrete series of line bundles over the unit ball of $\mathbb{C}^{d}$. Mich. Math. J. 45, 387-397 (1998)

[14] Rudin, W.: Function Theory in the Unit Ball of $\mathbb{C}^{n}$. Grundlehren der Math, vol. 241. Springer, New York (1980) 
[15] Shimeno, N.: The Plancherel formula for spherical functions with a onedimensional K-type on a simply connected simple Lie group of Hermitian type. J. Funct. Anal. 121, 330-388 (1994)

[16] Shimura, G.: On a class of nearly holomorphic automorphic forms. Ann. Math. 123, 347-406 (1986)

[17] Zhang, G.: A weighted Plancherel formula. II: the case of the ball. Stud. Math. 102, 103-120 (1992)

[18] Zhang, G.: Nearly holomorphic functions and relative discrete series of weighted $L^{2}$-spaces on bounded symmetric domains. J. Math. Kyoto Univ. 42, 207-221 (2002)

[19] Zheng, D.: Semi-commutators of Toeplitz operators on the Bergman space. Integr. Equ. Oper. Theory 25, 347-372 (1996)

[20] Zheng, D.: Commuting Toeplitz operators with pluriharmonic symbols. Trans. Am. Math. Soc. 350, 1595-1618 (1998)

[21] Zhu, K.: Operator Theory in Function Spaces. Operator Theory Advances \& Applications, 2nd edn. American Mathematical Society, Providence (2007)

Lijia Ding $(\otimes)$ and Kai Wang

School of Mathematical Sciences

Fudan University

Shanghai 200433

P.R. China

e-mail: 1 jding14@fudan.edu.cn

Kai Wang

e-mail: kwang@fudan.edu.cn

Received: May 16, 2018.

Revised: September 20, 2018. 